J. Asiat. Soc. Bangladesh, Sci. 39(2): 147-166, December 2013

\title{
ANGIOSPERM FLORA OF MANIKGONJ SADAR UPAZILA, BANGLADESH
}

\author{
KANIKA SARKER, MD. RAFIQUL ISLAM, MOHAMMAD ZASHIM UDDIN ${ }^{1}$ \\ AND MD. ABUL HASSAN \\ Department of Botany, University of Dhaka, Dhaka 1000, Bangladesh
}

\begin{abstract}
Angioperm flora of Manikgonj Sadar Upazila has been partially inventoried. A total of 207 species under 72 families and 174 genera has been recorded. Among those, the division Magnoliopsida represents 147 species belonging to 56 families and 127 genera, and the division Liliopsida represents 60 species belonging to 16 families and 47 genera.
\end{abstract}

Key words: Angiopermic flora; Manikgonj Sadar Upazila; Bangladesh

\section{Introduction}

The Sadar upazila of Manikgonj district occupies an area of $214.81 \mathrm{sq} . \mathrm{km}$. including $12.97 \mathrm{sq}$. $\mathrm{km}$. of river. The upazila lies between $23^{\circ} 42^{\prime}$ to $23^{\circ} 55^{\prime} \mathrm{N}$ latitudes and $89^{\circ} 58^{\prime}$ to $90^{\circ} 07^{\prime}$ E longitudes. It is bounded by Saturia upazila on the North, Nawabganj (Dhaka) and Harirampur upazilas on the South, Singair and Dhamrai upazilas on the East, and Harirampur and Ghior upazilas on the West. Main rivers of the upazila are the Dhaleshwari, the Ichamati, the Kaliganga and the Gazikhali (Ramzan 2003).

The Sadar upazila is mainly dominated by the extension of shallow upland soil. The soil of the area is mainly formed by the Brahmaputra river representing silty and sandy alluvial soil of moderate class. (Rizvi 1969). The area enjoys a tropical climate characterized by high precipitation from May to October and relatively dry period from November to April. The mean annual rainfall of the area is about $1671 \mathrm{~mm}$. Temperature of the area ranges from 19.5 to $33.9^{\circ} \mathrm{C}$. The maximum temperature oberved in May and minimum temperature observed in January. (Bangladesh Meteorological Department, personal communication).

A number of floristic works has so far been done in greater Dhaka district including Ismail and Mia (1973), Alam (1995), Hossain et al. (1995), Rahman and Hassan (1995), Rashid et al. (1995) and Alam et al. (2006). But no studies are found in Manikganj Sadar upazila. Moreover, the area supports a large number of angiopermic species including herbs, shrubs, trees, climbers, epiphytes, parasites and hydrophytes. Like other parts of the country, the floristic elements of this area are in risk because of various anthropogenic activities including irrigation, modern agriculture, population settlements, firewood collection, industrilization and also habitat degradation. In order to make a documentation of the angiospermic vegetation of the area an attempt has made to prepare a preliminary flora of the angiopermic plant species available at Manikganj Sadar upazila.

\footnotetext{
${ }^{1}$ Corresponding author: Email: suhavedu@yahoo.com
} 


\section{Materials and Methods}

This work is mainly based on the fresh plant materials collected by the author and the herbarium specimens collected from the same area and stored at different herbaria of the country. A standard survey method was developed to collect maximum number of plant species from all representative habitats found in the area. Four visits were made at three months interval during the year 2006 to collect specimens with either flowering and/or fruiting condition for recording seasonal variations. Those specimens were identified by matching with correctly identified herbarium specimens of Bangldesh National Herbarium and Dhaka University Salar Khan Herbarium (DUSH). In some cases, standard taxonomic literature [viz. Hooker (1872 - 1897), Prain (1903) and Uddin and Hassan (2004)] were also consulted to identify critical specimens. Voucher herbarium sheets were prepared for all species by using traditional herbarium technique and stored at DUSH."

The families have been arranged according to Cronquist (1981). The genera under each family and the species under each genus are arranged in an alphabetic order. For each species, nomenclature has been brought up-to-date; impotant synonyms, local name (wherever available) and a short annotation are also provided.

\section{Results and Discussion}

In the present survey, a total of 207 angiopermic species under 174 genera and 72 families were recorded from the Sadar upazila of Manikganj district. Magnoliopsida is represented by 56 families, 127 genera and 147 species, while Liliopsida is represented by 16 families, 47 genera and 60 species. Habit-wise itemization of plant species shows that $58 \%$ of the total species are represented by herbs, $21 \%$ by trees, $11 \%$ by shrubs, $10 \%$ by other groups (climbers, epiphytes and parasites).

\section{Magnoliopsida (Dicots)}

\section{Annonaceae}

Polyalthia longifolia (Sonn.) Thw., Enum. Pl. Zeyl.: 398 (1864). Uvaria longifolia Sonn. Local name: Debdaru. A tall tree. Representative specimens: Putail, 03.03.06, Kanika 42 (DUSH); Jaigir, 30.12.06, Kanika 241(DUSH).

P. suberosa (Roxb.) Thw., Enum. Pl. Zeyl.: 398 (1864). Uvaria suberosa Roxb. A shrub or small tree. Representative specimen: Putail, 15.06.06, Kanika 122 (DUSH).

Uvaria hamiltonii Hook. f. \& Thoms., Fl. Ind. 1: 96 (1820). A large climber.

\section{Lauraceae} Representative specimen: Nabagram, 04.03.06, Kanika 85 (DUSH).

Litsea monopetala (Roxb.) Pers., Syn. Pl. 2: 4 (1807). Tetranthera monopetala Roxb. Local names: Menda, Chapaitta. A medium-sized tree. Representative specimen: Manikgonj Sadar, 10.09.06, Kanika 154 (DUSH). 


\section{Piperaceae}

Peperomia pellucida (L.) Kunth. Nov. Gen. Sp. 1: 64 (1815). Piper pellucidum L. Local names: Luchipata, Peperomia. A small annual herb. Representative specimen: Putail, 11.09.06, Kanika 175 (DUSH).

Piper longum L., Sp. Pl. 1: 9 (1753). Local name: Pepul. A perennial climbing herb. Representative specimen: Putail, 03.03.06, Kanika 83 (DUSH).

\section{Aristolochiaceae}

Aristolochia tagala Cham., Linnaea 7: 207 (1832). Local name: Ishwarmul. A glabrous climber. Representative specimen: Jaigir, 16.06.06, Kanika 133 (DUSH).

\section{Nymphaeaceae}

Nymphaea nouchali Burm. f., Fl. Ind.: 120 (1768). Local names: Shapla, Kamal. A perennial aquatic herb. Representative specimens: Betila, 12.09.06, Kanika 194 (DUSH); Jaigir, 30.12.06, Kanika 242 (DUSH).

N. rubra Roxb. ex Andr., Bot. Rep. 8: 104, t. 503 (1808). Local names: Lal-Shapla, Ogul phul. A perennial aquatic herb. Representative specimen: Manikgonj Sadar, 02.03.06, Kanika 17 (DUSH).

\section{Ranunculaceae}

Ranunculus sceleratus L., Sp. Pl.: 551 (1753). An erect, annual herb. Representative specimen: Manikgonj Sadar, 02.03.06, Kanika 35 (DUSH).

\section{Menispermaceae}

Stephania japonica (Thunb.) Miers, Ann. Mag. Nat. Hist. Ser. 3, 18: 14 (1866). Menispermum japonicum Thunb. Local names: Aknadi, Maknadi, Nimukha. A slender climber. Representative specimen: Betila, 15.06.06, Kanika 127 (DUSH).

Tinospora sinensis (Lour.) Merr., Sunyatsenia 1: 193 (1934). Campylus sinensis Lour. Local name: Gulonchoe. A climbing shrub. Representative specimen: Manikgonj Sadar, 02.03.06, Kanika 01(DUSH).

\section{Papaveraceae}

Argemone mexicana L., Sp. Pl. 1: 508 (1753). Local name: Sialkanta. An annual herb. Representative specimen: Manikgonj Sadar, 02.03.06, Kanika 36 (DUSH).

\section{Ulmaceae}

Trema orientalis (L.) B1., Mus. Bot. Lugd.-Bat. 2: 62 (1856). Celtis orientalis L. Local names: Jilan, Chikan, Nars. An ever green, small tree. Representative specimen: Manikgonj Sadar, 02.03.06, Kanika 37 (DUSH).

\section{Moraceae}

Artocarpus lacucha Buch.-Ham., Mem. Wern. Soc. 5: 333 (1826). Local name: Dephul. A large deciduous tree. Representative specimen: Garpara, 28.12.06, Kanika 222 (DUSH). 
Ficus benghalensis L., Sp. Pl. 1: 1059 (1753). A large tree. Representative specimen: Nabagram, 04.03.06, Kanika 86 (DUSH).

F. heterophylla L. f., Suppl. Pl.: 442 (1781). A scandent shrub. Representative specimen: Putail, 03.03.06, Kanika 43 (DUSH).

F. hispida L. f., Suppl. Pl.: 442 (1781). Local name: Kakdumur. A low tree. Representative specimen: Putail, 11.09.06, Kanika 176 (DUSH).

F. religiosa L., Sp. Pl.: 1059 (1753). Local names: Aswatha, Pakur. A large tree. Representative specimen: Garpara, 17.06.06, Kanika 144 (DUSH).

\section{Urticaceae}

Laportea interrupta (L.) Chew, Gard. Bull. Singapore. 21: 200 (1965). Urtica interrupta L. Local name: Lal Bichuti. A shrub. Representative specimen: Putail, 15.06.06, Kanika 123 (DUSH).

Pilea microphylla (L.) Liebm., Vidensk. Selsk. Skr. 5, Ser: 2: 302 (1851). Parietaria microphylla L. An annual, prostrate herb. Representative specimen: Manikgonj Sadar, 10.09.06, Kanika 155 (DUSH).

Pouzolzia zeylanica (L.) Benn., Pl. Jav. Rar.: 67 (1833). Parietaria zeylanica L. An erect or trailing herb. Representative specimen: Betila, 15.06.06, Kanika 128 (DUSH).

\section{Nyctaginaceae}

Boerhaavia diffusa L., Sp. Pl. 1: 3 (1753). Local name: Punarnava. A perennial procumbent herb. Representative specimen: Putail, 03.03.06, Kanika 82 (DUSH).

\section{Chenopodiaceae}

Chenopodium album L., Sp. PI. 1: 219 (1753). Local name: Batua Shak. An erect, annual herb. Representative specimen: Nabagram, 27.12.06, Kanika 206 (DUSH).

C. ambrosioides L., Sp. Pl. 1: 219 (1753). An erect, annual herb. Representative specimen: Jaigir, 16.06.06, Kanika 134 (DUSH).

\section{Amaranthaceae}

Achyranthes aspera L., Sp. Pl. 1: 204 (1753). Local name: Apang. A perennial herb. Representative specimen: Garpara, 28.12.06, Kanika 223 (DUSH).

Alternanthera philoxeroides (Mart.) Griseb., Symb. Argent. in Abh. Ges. Wiss. Gott. 24: 36 (1879). Bucholzia philoxeroides Mart. Local name: Helencha Sak. A perennial or annual herb. Representative specimen: Nabagram, 04.03.06, Kanika 102 (DUSH).

A. sessilis (L.) A. Dc., Cat. Pl. Horti Monsp.:77 (1813). Gomphrena sessilis L. Local names: Chanchi, Haicha, Sachisak. An annual or perennial, prostrate herb. Representative specimen: Putail, 15.06.06, Kanika 124 (DUSH). 
Angiosperm flora of Manikgonj

A. spinosus L., Sp. P1. 1: 991 (1753). Local name: Kantanotey, Kantadenga, Katamiris. An annual, erect, spinescent herb. Representative specimen: Betila, 12.09.06, Kanika 195 (DUSH).

A. viridis L., Sp. Pl. ed. 2: 1405 (1753). Local names: Marissag, Notey, Notey shak. An annual, erect herb. Representative specimen: Manikgonj Sadar, 02.03.06, Kanika 02 (DUSH).

\section{Portulacaceae}

Portulaca oleracea L., Sp. Pl. 1: 445 (1753). Local names: Bara Lanya, Bara Nunia. A prostrate annual herb. Representative specimen: Putail, 03.03.06, Kanika 44 (DUSH).

\section{Polygonaceae}

Persicaria hydropiper (L.) Spach., Hist. Nat. Veg. 10: 536 (1841). Polygonum hydropiper L. Local name: Pakurmul. An annual herb. Representative specimens: Manikgonj Sadar, 10.09.06, Kanika 156 (DUSH); Garpara, 28.12.06, Kanika 224 (DUSH).

Polygonum effusum Meissn. in DC., Prodr. 14: 93 (1857). Polygonum plebeium R.Br. var effusum Hook. f. A much-branched, prostrate annual herb. Representative specimens: Jaigir, 30.12.06, Kanika 261(DUSH).

Rumex maritimus L., Sp. Pl. 1: 335 (1753). Local name: Bon-palong. An annual erect herb. Representative specimen: Betila, 15.06.06, Kanika 129 (DUSH).

\section{Dilleniaceae}

Dillenia indica L., Sp. Pl.: 535 (1753). Local name: Chalta. A medium sized tree. Representative specimen: Jaigir, 30.12.06, Kanika 259 (DUSH).

\section{Tiliaceae}

Grewia nervosa (Lour) G. Panigrahi, Taxon 34 (2): 702 (1985). Microcos paniculata L. Local names: Asar, Assar, Patka, Dattoi. A shrub or small tree. Representative specimen: Manikgonj, 02.03.06, Kanika 18 (DUSH).

\section{Sterculiaceae}

Abroma augusta (L.) L. f., Suppl. Pl.: 341 (1781). Theobroma augusta L. Local name: Ulatkambal. A shrub. Representative specimen: Putail, 11.09.06, Kanika 178 (DUSH).

\section{Bombacaceae}

Bombax ceiba L., Sp. Pl.: 511 (1753). Local name: Shimul. A large tree with buttress base. Representative specimen: Putail, 03.03.06, Kanika 81 (DUSH).

\section{Malvaceae}

Abelmoschus moschatus Medic., Malv.: 46 (1787). A tuberous, prickly herb. Representative specimen: Nabagram, 27.12.06, Kanika 207 (DUSH). 
Abutilon indicum (L.) Sweet., Hort. Brit. ed. 1: 54 (1826). Sida indica L. An annual or perennial herb. Representative specimen: Putail, 03.03.06, Kanika 84 (DUSH).

Sida cordata (Burm. f.) van Boriss., Blumea 14 (1): 182 (1966). Melochia cordata Burm. f. Local names: Junka, Sunate. An annual, prostrate or ascending herb. Representative specimen: Nabagram, 04.03.06, Kanika 87 (DUSH).

\section{Lecythidaceae}

Barringtonia acutangula (L.) Gaertn., Fruct. 2: 97, t. 101 (1791). Eugenia acutangula L. A medium-sized tree. Representative specimen: Jaigir, 30.12.06, Kanika 265 (DUSH).

Careya arborea Roxb., Pl. Corom. 3: 14, t. 218 (1811). Local names: Kumba, Gade. A low tree. Representative specimen: Manikgonj Sadar, 10.09.06, Kanika 160 (DUSH).

\section{Cucurbitaceae}

Coccinia grandis (L.) Voigt, Hort. Suburb. Calcut.: 59 (1845). Bryonia grandis L. Local name: Telakucha. A climber. Representative specimen: Nabagram, 04.03.06, Kanika 103 (DUSH).

Mukia maderaspatana (L.) M. Roem., Fam. Nat. Syn. Monogr. 2: 47 (1846). Local name: Bilari. A perennial, climbing herb. Representative specimens: Putail, 03.03.06, Kanika 45 (DUSH); Garpara, 28.12.06, Kanika 226 (DUSH).

\section{Capparaceae}

Cleome viscosa L., Sp. Pl. 2: 672 (1753). An erect, annual herb. Representative specimen: Betila, 12.09.06, Kanika 196 (DUSH).

Crataeva magna (Lour.) DC., Prodr. 1: 243 (1824). Capparis magna Lour. Local name: Barun. Deciduous tree. Representative specimen: Putail, 03.03.06, Kanika 80 (DUSH).

\section{Brassicaceae}

Rorippa indica (L.,) Hiern, Cat. Afr. Pl. Welw.1: 26 (1896). Sisymbrium indicum L. An annual herb. Representative specimen: Jaigir, 16.06.06, Kanika 135 (DUSH).

\section{Sapotaceae}

Mimusops elengi L., Sp. P1.: 349 (1753). Local name: Bakul. A large evergreen tree. Representative specimen: Putail, 15.06.06, Kanika 125 (DUSH).

\section{Ebenaceae}

Diospyros montana Roxb., Pl. Corom. 1: 37 (1795). Local name: Gab. A small tree. Representative specimen: Putail, 03.03.06, Kanika 46 (DUSH).

\section{Mimosaceae}

Acacia nilotica (L.) Delile subsp. indica (Benth.) Brenan in Kew Bull. 12: 84 (1957). Mimosa nilotica L. Local name: Babla. A tree. Representative specimen: 
Nabagram, 04.03.06, Kanika 101 (DUSH).

Albizzia procera Benth. in Hook., London J. Bot. 3: 89 (1844). Local names: Koroi, Jat Koroi, Sada Koroi, Sil Koroi. A medium-sized tree. Representative specimen: Garpara, 17.06.06, Kanika 146 (DUSH).

Mimosa pudica L., Sp. Pl.: 518 (1753). Local names: Lojjabati, Lajak. A prickly, woody herb. Representative specimens: Nabagram, 04.03.06, Kanika 87 (DUSH); Garpara, 28.12.06, Kanika 228 (DUSH).

Pithecellobium angulatum Benth. in Hook., London J. Bot. 3: 208 (1844). A tree. Representative specimen: Manikgonj Sadar, 02.03.06, Kanika 38 (DUSH).

\section{Caesalpiniaceae}

Cassia fistula L., Sp. Pl: 377 (1753). Local name: Badarlathi. A small tree. Representative specimen: Nabagram, 27.12.06, Kanika 208 (DUSH).

Senna sophera (L.) Roxb., Fl. Ind. 2: 347 (1832). Cassia sophera L. Local names: Chotokalkesunda, Jhingi. A shrub or undershrub. Representative specimen: Manikgonj Sadar, 02.03.06, Kanika 34 (DUSH).

S. tora (L.) Roxb., Fl. Ind. 2: 340 (1832). Cassia tora L. A perennial, erect herb or undershrub. Representative specimen: Jaigir, 30.12.06, Kanika 259 (DUSH).

\section{Fabaceae}

Butea monosperma (Lamk.) Taub. in Engl. \& Prantl, Nat. Pflanz. 3(3): 366 (1894). Erythrina monosperma Lamk. Local name: Palash. A deciduous tree. Representative specimen: Putail, 03.03.06, Kanika 79 (DUSH).

Cajanus cajan (L.) Druce, Rep. Bot. Exch. Cl. Brit. Isles, 1916: 611 (1917). Cytisus cajan L. Local name: Orhor. A shrub. Representative specimen: Nabagram, 04.03.06, Kanika 100 (DUSH).

Crotalaria pallida Aiton, Hort. Kew. 2: 20 (1789). Local name: Bara jhunjhuni. An annual herb. Representative specimen: Jaigir, 30.12.06, Kanika 266 (DUSH).

Dalbergia sissoo Roxb., Fl. Ind. 3: 223 (1832). Local name: Sisso. A tree. Representative specimens: Nabagram, 04.03.06, Kanika 104 (DUSH); Garpara, 28.12.06, Kanika 229 (DUSH).

Desmodium hetrophyllum (Willd.) DC., Prodr. 2: 334 (1825). Hedysarum heterophyllum Willd. A small herb. Representative specimen: Garpara, 28.12.06, Kanika 230 (DUSH).

D. triflorum (L.) DC., Prodr. 2:334 (1825). Hedysarum triflorum L. A trailing herb. Representative specimen: Putail, 03.03.06, Kanika 47 (DUSH).

D. triquetrum (L.) DC., Prodr. 2: 326 (1825). Hedysarum triquetrum L. A shrub. Representative specimen: Jaigir, 30.12.06, Kanika 255 (DUSH).

Erythrina ovalifolia Roxb., Fl. Ind. 3: 251 (1832). Local name: Mandar. A small deciduous tree. Representative specimen: Jaigir, 30.12.06, Kanika 254 (DUSH). 
E. variegata L., Diss. Herb. Amb. Amoen. Acad. 4: 122 (1754). A small to medium sized tree. Representative specimen: Betila, 15.06.06, Kanika 130 (DUSH).

Sesbania grandiflora (L.) Poir. in Lamk., Encycl. Met. 7: 127 (1806). Aeschynomene grandiflora L. A tree. Representative specimen: Nabagram, 04.03.06, Kanika 88 (DUSH).

\section{Lythraceae}

Lagerstroemia speciosa (L.) Pers., Syn. 2: 72 (1807). Munchausia speciosa L. Local name: Jarul. A large, deciduous tree. Representative specimen: Garpara, 17.06.06, Kanika 147 (DUSH).

\section{Myrtaceae}

Syzygium fruticosum (Roxb.) DC., Prodr. 3: 260 (1828). Eugenia fruticosa Roxb. Local names: Banjam, Khudijam. A small tree. Representative specimen: Nabagram, 04.03.06, Kanika 99 (DUSH).

\section{Onagraceae}

Ludwigia adscendens (L.) Hara, J. Jap. Bot. 28: 291 (1953). Jussiaea abyssinica L. Local name: Keshardam. A creeping aquatic herb. Representative specimen: Manikgonj Sadar, 10.09.06, Kanika 161 (DUSH).

L. hyssopifolia (G. Don) Exell., Fernand. Garica de Orta 5: 471 (1957). Jussiaea hyssopifolia G. Don. A herb. Representative specimen: Jaigir, 16.06.06, Kanika 136 (DUSH).

\section{Combretaceae}

Terminalia arjuna (Roxb. ex DC.) Wight. \& Arn., Prodr.: 314 (1834). Pentaptera arjuna Roxb. ex DC. Local name: Arjun. A medium-sized tree. Representative specimen: Nabagram, 27.12.06, Kanika 209 (DUSH).

T. citrina (Gaertn.) Roxb. ex Fleming, Asiat. Res. 11: 183 (1810). Myrobalanus citrina Gaertn. Local name: Horitaki. A tall tree. Representative specimen: Jaigir, 30.12.06, Kanika 243 (DUSH).

\section{Loranthaceae}

Dendrophthoe falcata (L. f.) Etting., Denkschr. Akad. Wisscnch. Wicn. Math.- Natur. Cl. 32: 51 (1872). Loranthus falcatus L. f. Local names: Bancha, Phorolla. A parasite shrub. Representative specimen: Manikgonj, 02.03.06, Kanika16 (DUSH).

\section{Euphorbiaceae}

Croton bonplandianus Bill., Adansonia 4: 339 (1864). Local name: Moricha. An annual herb. Representative specimen: Jaigir, 30.12.06, Kanika 257 (DUSH).

Euphorbia hirta L., Sp. Pl.: 454 (1753). Local names: Ghaopata, Dudhia. An annual, robust herb. Representative specimen: Putail, 11.09.06, Kanika 180 (DUSH). 
Jatropha curcas L., Sp. Pl. 2: 1006 (1753). A shrub or small tree. Representative specimen: Manikgonj Sadar, 02.03.06, Kanika 19 (DUSH).

J. gossypifolia L., Sp. P1. 2: 1006 (1753). Local names: Lalbherenda, Laljeol. A small shrub. Representative specimen: Nabagram, 04.03.06, Kanika 105 (DUSH).

Macaranga denticulata (Blume) Muell.-Arg. in DC., Prodr. 15 (2): 1000 (1886). Mappa denticulata Blume. Local name: Bura. A small, ever green tree. Representative specimen: Garpara, 17.06.06, Kanika 145 (DUSH).

Phyllanthus reticulatus Poir. in Lamk., Encycl. Meth. B. 5: 298 (1804). Local names: Panseuli, Panku. A large, scandent shrub. Representative specimen: Manikgonj Sadar, 02.03.06, Kanika 39 (DUSH).

Ricinus communis L., Sp. Pl. 2, 1007 (1753). Local name: Redhi. An ever green shrub. Representative specimen: Putail, 03.03.06, Kanika 49 (DUSH).

Trewia nudiflora L., Sp. P1.: 2, 1193 (1753). Local names: Bhatam, Lattu, Latim. Pitali. A medium-sized deciduous tree. Representative specimen: Nabagram, 04.03.06, Kanika 89 (DUSH).

\section{Vitaceae}

Cayratia trifolia (L.) Domin, Biblioth. Bot. 89: 370 (1927). Vitis trifolia L. Local name: Amal-lata. A herbaceous climber. Representative specimen: Nabagram, 27.12.06, Kanika 210 (DUSH).

Cissus quadrangularis L., Syst. Nat. ed. 12 (2): 124 (1767). Local names: Harjora lata, Kumor lata. A herbaceous climber. Representative specimen: Jaigir, 30.12.06, Kanika 267 (DUSH).

\section{Anacardiaceae}

Lannea coromandelica (Houtt.) Merr., J. Arnold Arbor. 19: 353 (1938). Dialium coromandelicum Houtt. Local names: Jiga, Jeol, Jika, Bhadi. A medium-sized, deciduous tree. Representative specimen: Putail, 03.03.06, Kanika 78 (DUSH).

\section{Meliaceae}

Aphanamixis polystachya (Wall.) Parker, Ind. For. 57: 486 (1931). Aglaia polystachya Wall. Local names: Royna, Roonna, Pitraj. A tree. Representative specimen: Jaigir, 30.12.06, Kanika 256 (DUSH).

Azadirachta indica A. Juss., Mem. Mus. Hist. Nat. Paris 19: 221, t. 13 (1830). Local name: Neem. An evergreen tree. Representative specimen: Putail, 11.09.06, Kanika 181 (DUSH).

Melia azedarach L., Sp. Pl.: 384 (1753). Local names: Mohanim, Bokain, Poma, Ghora neem. A medium-sized cultivated tree. Representative specimen: Manikgonj, 02.03.06, Kanika 33 (DUSH). 


\section{Rutaceae}

Aegle marmelos (L.) Corr., Trans. Linn. Soc. 5: 223 (1800). Crataeva marmelos L. Local name: Bel. A small, deciduous tree. Representative specimen: Nabagram, 04.03.06, Kanika 106 (DUSH). Cultivated.

Glycosmis pentaphylla (Retz.) A. DC., Prodr. 1: 538 (1824). Limonia pentaphylla Retz. Local names: Datmajan, Matkila, Ranggach, Matmati. A shrub or small tree. Representative specimen: Nabagram, 27.12.06, Kanika 211 (DUSH).

Limonia acidissima L., Sp. Pl. ed. 1: 554 (1763). Limonia elephantum (Correa) Panigrah. Local name: Kathbel. A medium-sized, semi-deciduous, spiny tree. Representative specimen: Putail, 03.03.06, Kanika 50 (DUSH).

\section{Oxalidaceae}

Averrhoa carambola L., Sp. Pl.1: 428 (1753). Local name: Kamranga. A bushy tree. Representative specimen: Nabagram, 04.03.06, Kanika 90 (DUSH).

Oxalis corniculata L., Sp. Pl. 1: 435 (1753). Local names: Amrul, Amboli, Chukatripati. An annual herb. Representative specimen: Putail, 15.06.06, Kanika 126 (DUSH).

\section{Apiaceae}

Centella asiatica (L.) Urban in Mart., Fl. Bras. 11: 287 (1879). Hydrocotyle asiatica L. Local names: Thankuni, Thulkuri, Brahmabuti. A perennial herb. Representative specimen: Jaigir, 16.06.06, Kanika 137 (DUSH).

\section{Apocynaceae}

Alstonia scholaris (L.) R. Br., Mem. Wern. Nat. Hist. Soc. 1: 75 (1811). Echites scholaris L. Local names: Chhatim, Chatwan. A medium-sized tree. Representative specimen: Jaigir, 30.12.06, Kanika 250 (DUSH).

Ichnocarpus frutescens (L.) R. Br. in Mem. Wern. Soc. 1: 62 (1809). Apocynum frutescens L. Local names: Dudhilata, Kalilata, Loi, Paralilata, Shamalata. A climbing shrub. Representative specimen: Manikgonj Sadar, 02.03.06, Kanika 39 (DUSH).

Rauwolfia serpentina (L.) Benth. ex Kurz, For. Fl. Brit. Burma 2: 171 (1877). Ophioxylon serpentinum L. Local name: Sarpaganda. A woody herb. Representative specimen: Garpara, 17.06.06, Kanika (DUSH).

\section{Asclepiadaceae}

Calotropis gigantea (L.) W.T. Aiton, Hort. Kew. ed. 2, 2: 78 (1811). Aselepias gigantea L. A large shrub. Representative specimen: Putail, 03.03.06, Kanika 77 (DUSH).

C. procera (Ait.) R. Br. in Ait. f., Hort. Kew. ed. 2, 2:78 (1811). Asclepias procera Ait. Local name: Akanda. A large shrub. Representative specimen: Garpara, 17.06.06, Kanika 148 (DUSH). 


\section{Solanaceae}

Datura metel L., Sp. P1.: 179 (1753). Local name: Dutra. A pernnial herb. Representative specimen: Jaigir, 16.06.06, Kanika 137 (DUSH).

Nicotiana plumbaginifolia Viv., Elench. Pl. Hort. Dinegro: 26. t. 5 (1802). Local name: Ban-tamak. An erect, annual herb. Representative specimen: Nabagram, 27.12.06, Kanika 212 (DUSH).

Physalis minima L., Sp. Pl.: 183 (1753). Local names: Byakur, Gurkamai. An annual herb. Representative specimen: Jaigir, 30.12.06, Kanika 268 (DUSH).

Solanum nigrum L., Sp. Pl.: 186 (1753). Local name: Tit begun. An annual shrub. Representative specimen: Putail, 03.03.06, Kanika 51 (DUSH).

S. torvum Sw., Nov. Gen. Sp. Pl.: 47 (1788). Local name: Bot begun. A small shrub. Representative specimen: Betila, 12.09.06, Kanika 198 (DUSH).

\section{Convolvulaceaess}

Ipomoea aquatica Forssk., Fl. Aeg. Arab.: 44 (1775). Local name: Kalmilata. An aquatic herb. Representative specimen: Nabagram, 04.03.06, Kanika 108 (DUSH).

I. fistulosa Mart. ex Choisy in DC., Prodr. 9: 349 (1845). Local names: Dholkalmi, Durakalma. A shrub. Representative specimen: Jaigir, 30.12.06, Kanika 269 (DUSH).

Merremia hederacea (Burm. f.) Hallier f., Bot. Jahrb. 18: 118 (1994). Evolvulus hederaceus Burm. f. A twinner. Representative specimen: Garpara, 17.06.06, Kanika 149 (DUSH).

M. hirta (L.) Merr., Philip. J. Sc. Bot. 7: 244 (1912). Convolvulus hirtus L. A slender twiner. Representative specimen: Putail, 03.03.06, Kanika 76 (DUSH).

\section{Cuscutaceae}

Cuscuta reflexa Roxb., P1. Corom. 2: 3, t. 104 (1798). Local names: Algusi, Jarbuti, Swarnalata. A fleshy parasite. Representative specimen: Nabagram, 27.12.06, Kanika 211 (DUSH).

\section{Boraginaceae}

Heliotropium indicum L., Sp. Pl.: 139 (1753). Local name: Hatisur. An annual herb. Representative specimen: Manikgonj Sadar, 10.09.06, Kanika 165 (DUSH).

\section{Verbenaceae}

Clerodendrum indicum (L.) Kuntze, Rev. Gen. Pl. 2: 586 (1891). Siphonanthus indica L. Local names: Bamunhati, Banchat. An undershrub. Representative specimen: Manikgonj Sadar, 02.03.06, Kanika 31 (DUSH).

C. viscosum Vent., Jard. Malm. 1: t. 25 (1803). Local names: Bhant, Ghetu, Ghetuphul. A perennial, woody herb. Representative specimen: Putail, 11.09.06, Kanika 191 (DUSH). 
Duranta repens L., Sp. P1. 1: 637 (1753). Local names: Duranta, Kantamehedi. A shrub or small tree. Representative specimens: Nabagram, 04.03.06, Kanika 109 (DUSH); Garpara, 28.12.06, Kanika 231 (DUSH).

Lantana camara L., Sp. P1.: 627 (1753). A shrub. Representative specimens: Putail, 11.09.06, Kanika 183 (DUSH); Garpara, 28.12.06, Kanika 232 (DUSH).

Lippia javanica (Burm. f.) Spreng., Syst. 2: 752 (1825). An undershrub. Representative specimen: Jaigir, 30.12.06, Kanika 270 (DUSH).

.Vitex pubescens Vahl, Symb. Bot. 3: 85 (1794). A large tree. Representative specimen: Manikgonj Sadar, 02.03.06, Kanika 40 (DUSH).

\section{Lamiaceae}

Anisomeles indica (L.) O. Kuntze, Rev. Gen.: 512 (1891). Nepeta indica L. Local name: Gobura. A bushy undershrub. Representative specimen: Putail, 03.03.06, Kanika 52 (DUSH).

Dysophylla crassicaulis Benth. in Wall., Pl. As. Rar. 1: 30 (1830). An annual herb. Representative specimen: Garpara, 17.06.06, Kanika (DUSH).

Leonurus sibiricus L., Sp. Pl.: 584 (1735). An erect sherb. Representative specimen: Nabagram, 04.03.06, Kanika (DUSH).

Leucas aspera Spreng., Syst. 2: 743 (1825). Local name: Durung pata. An annual herb. Representative specimen: Jaigir, 30.12.06, Kanika 251 (DUSH).

Ocimum tenuiflorum L., Sp. Pl.: 597 (1753). Ocimum sanctum L. Local name: Kalo tulshi. A much branched, soft hairy, perennial herb. Representative specimen: Betila, 12.09.06, Kanika 199 (DUSH).

\section{Scrophulariaceae}

L. crustacea (L.) F. Muell., Census Austral. P1. 1: 97 (1882). Capraria crustacea L. A small annual herb. Representative specimen: Putail, 15.06.06, Kanika 127 (DUSH).

Scoparia dulcis L., Sp. Pl.: 166 (1753). Local names: Bandhuni, Bandhoney, Phurphuri. A herb. Representative specimen: Manikgonj Sadar, 02.03.06, Kanika 30 (DUSH).

\section{Acanthaceae}

Andrographis paniculata (Burm.f.) Nees in Wall., P1. Asiat. Rar. 3: 116 (1832). Justicia paniculata Burm. f. Local name: Kalmegh. An erect annual herb. Representative specimen: Putail, 03.03.06, Kanika 63 (DUSH).

Justicia adhatoda L., Sp. Pl.: 15 (1753). Adhatoda vasica Nees. Local name: Basak. A shrub. Representative specimens: Nabagram, 04.03.06, Kanika 91 (DUSH); Jaigir, 30.12.06, Kanika 245 (DUSH).

J. gendarussa Burm. f., Fl. Ind.: 10 (1768). Local names: Jagatmadan, Jagmadan. An undershrub. Representative specimen: Jaigir, 30.12.06, Kanika 248 (DUSH). 
Angiosperm flora of Manikgonj

Rungia pectinata (L.) Nees in DC., Prodr. 11: 470 (1847). Justicia pectinata L. Local name: Pindi. A prostrate or suberect herb. Representative specimen: Manikgonj Sadar, 02.03.06, Kanika 15 (DUSH).

\section{Bignoniaceae}

Fernandoa adenophyllla (Wall. ex. G. Don.) van Steenis, Blumea 23: 135 (1976). Bignonia adenophylla Wall. ex. G. Don. Local name: Banpata. A tree. Representative specimen: Nabagram, 04.03.06, Kanika 110 (DUSH).

\section{Campanulaceae}

Lobelia radicans Thunb., Trans. Linn. Soc. 2: 330 (1794). A prostrate herb. Representative specimen: Putail, 03.03.06, Kanika 53 (DUSH).

\section{Rubiaceae}

Dentella repens (L.) J. R. \& G. Forst., Char. Gen. P1. Ins. Mar. Austr.: 26, t, 13 (1776). Oldenlandia repens L. Local name: Bhuipat. An annual prostrate herb. Representative specimen: Manikgonj Sadar, 02.03.06, Kanika 20 (DUSH).

Ixora acuminata Roxb., Fl. Ind. 1: 393 (1820). An undershrub. Representative specimen: Nabagram, 27.12.06, Kanika 212 (DUSH).

Neolamarckia cadamba (Roxb.) Bosser, Bull. Mus. Hist. Nat. Paris, Ser. 6, Sec. B, Adans. 3: 247 (1984). Anthocephalus cadamba (Roxb.) miq.; Anthocephalus chinensis (Lamk.) A. Rich. ex walk. Local name: Kadam. A large tree spreading sub whorled branches. Representative specimen: Putail, 03.03.06, Kanika 64 (DUSH).

Paederia foetida L., Mant. Pl. 1: 52 (1767). Local name: Gandha badhuli. A slender climber. Representative specimen: Nabagram, 04.03.06, Kanika 111 (DUSH).

\section{Asteraceae}

Ageratum conyzoides L., Sp. Pl.: 839 (1753). Local names: Dulkuri, Hialmuti. An annual herb. Representative specimen: Manikgonj Sadar, 10.09.06, Kanika 166 (DUSH).

Blumea lacera (Burm. f.) DC. in Wight, Contrib. Bot. Ind.: 14 (1834). Conyza lacera Burm. f. Local names: Barakukshima, Barakosing, Barasaksang, Kukursunga, Kuksung. An erect, annual herb. Representative specimen: Nabagram, 04.03.06, Kanika 92 (DUSH).

Chromolaena odorata (L.) King \& Robinson, Phytologia 20: 204 (1970). Eupatorium odoratum L. Local names: Germanlata, Barashialmuti. An erect herb. Representative specimen: Jaigir, 30.12.06, Kanika 252 (DUSH).

Eclipta alba (L.) Hassk., Pl. Jav. Rar.: 528 (1848). Local name: Kesoraj. An annual herb. Representative specimen: Manikgonj Sadar, 02.03.06, Kanika 04 (DUSH).

Enhydra fluctuans Lour., Fl. Cochinch.: 511 (1790). Local name: Helencha. An annual aquatic herb. Representative specimen: Putail, 03.03.06, Kanika 54 (DUSH). 
Gnaphalium luteo-album L., Sp. Pl.: 851 (1753). Local name: Bara kamra. An erect, annual herb. Representative specimen: Jaigir, 30.12.06, Kanika 247(DUSH).

Grangea maderaspatana (L.) Poir., Enc. Suppl. 2: 825 (1811). Artemisia maderaspatana L. Local name: Nemuti. An annual herb. Representative specimen: Manikgonj, 02.03.06, Kanika 14 (DUSH).

Mikania cordata (Burm. f.) B.L. Robinson, Contrib. Gray Herb. 104: 65 (1934). Eupatorium cordatum Burm. f. Local names: Assamlata, Tarulata. A perennial herb. Representative specimen: Putail, 03.03.06, Kanika 69 (DUSH).

Spilanthes calva DC. in Wight, Contrib. Bot. Ind.: 19 (1834) Spilanthes acmella auct. non L. Local name: Marhatitiga. An annual herb. Representative specimen: Putail, 03.03.06, Kanika 70 (DUSH).

Synedrella nodiflora (L.,) Gaertn., Fruct. 2: 456. t. 171 (1791). An erect annual herb. Representative specimen: Jaigir, 16.06.06, Kanika 140 (DUSH).

Tridax procumbens L., Sp. Pl.: 900 (1753). Local name: Tridara. An annual or perennial, procumbent herb. Representative specimen: Betila, 12.09.06, Kanika 200 (DUSH).

Vernonia cinerea (L.) Less., Linnaea 4(1): 291 (1829). Conyza cinerea L. Local name: Shial lata. An erect herb. Representative specimen: Putail, 03.03.06, Kanika 55 (DUSH).

Wedelia chinensis (Osbeck) Merr., Philipp. J. Sci. Bot. 12: 111(1917). Solidago clinensis Osbeck. Local name: Kesharaja. A procumbent or decumbent herb. Representative specimen: Putail, 03.03.06, Kanika 65 (DUSH).

Xanthium strumarium L., Sp. Pl. 2: 987 (1753). Local name: Gaghra. An erect annual herb. Representative specimen: Manikgonj Sadar, 02.03.06, Kanika 21 (DUSH).

\section{Liliopsida (Monocots)}

\section{Alismataceae}

Sagittaria guayanensis H.B. \& K. subsp. lappula (D. Don) Bogin in Mem. N.Y. Bot.,Gard. 9: 192 (1955). Sagittaria guayanensis sensu Hook. An annual, scapigerous herb. Representative specimen: Nabagram, 27.12.06, Kanika 213 (DUSH).

S. sagittifolia L., Sp. P1. 2: 993 (1753). Local names: Muyamuya, Chhotokut. A scapigerous aquatic herb. Representative specimen: Putail, 11.09.06, Kanika 185 (DUSH).

\section{Hydrocharitaceae}

Blyxa octrandra (Roxb.) Planch. ex. Thw. Enum. Pl. Zeyl.: 332 (1864). A stemless, submerged herb. Representative specimen: Nabagram, 04.03.06, Kanika 97 (DUSH).

Hydrilla verticillata (L. f.) Royle, 3. Bot. Himal. t. 376. (1839). Serpicula verticillata L. f. An auquatic herb. Representative specimen: Putail, 03.03.06, Kanika 68 (DUSH). 
Ottelia alismoides (L.) Pers., Syn. P1. 1: 400 (1805). Stratiotes alismoides L. A submerged herb. Representative specimens: Manikgonj Sadar, 02.03.06, Kanika 05 (DUSH); Garpara, 28.12.06, Kanika 234 (DUSH).

\section{Aponogetonaceae}

Aponogeton appendiculatus van Bruggen in Blumea 16: 265 (1968). Local name: Gheehu. A perennial stoloniferous herb. Representative specimen: Manikgonj Sadar, 02.03.06, Kanika 29 (DUSH).

A. natans (L.) Engl. \& Krause, Pflanzenreich, Aponogetonaceae: 11 (1906). Saururus natans L. A herb with stoloniferous rootstock. Representative specimen: Nabagram, 27.12.06, Kanika 214 (DUSH).

\section{Arecaceae}

Calamus viminalis Willd, Sp. Pl. 2 (1): 203 (1799). A thicket forming climbers. Representative specimen: Putail, 11.09.06, Kanika 189 (DUSH).

Caryota urens L., Sp. Pl.: 1189 (1753). An erect, solitary palm. Representative specimens: Putail, 03.03.06, Kanika 66 (DUSH); Garpara, 28.12.06, Kanika 235 (DUSH).

\section{Araceae}

Alocasia macrorrhizos (L) G. Don in Sweet, Hort. Brit. ed. 3: 631(1839). Arum macrorrhizon L. Local name: Man kachhu. A stout herb. Representative specimen: Manikgonj Sadar, 02.03.06, Kanika 06 (DUSH).

Amorphophallus bulbifer (Roxb.) Blume in Rumphia 1: 148 (1837). Arum bulbiferum. Roxb. A tuberous herb. Representative specimen: Manikgonj Sadar, 10.09.06, Kanika 167 (DUSH).

Colocasia esculenta (L.) Schott in Schott \& Endl., Melet. Bot.: 18 (1832). Arum esculenta L. A perennial herb. Representative specimen: Putail, 03.03.06, Kanika 56 (DUSH).

Lasia spinosa (L.) Thw., Enum. P1. Zeyl.: 336 (1864). Dracontium spinosum L. Local name: Kanta kanchhu. A prickly, aquatic herb. Representative specimen: Nabagram, 04.03.06, Kanika 93 (DUSH).

Pistia stratiotes L., Sp. Pl. 963 (1753). A free floating, monoecious herb. Representative specimen: Manikgonj Sadar, 02.03.06, Kanika 23 (DUSH).

T. trilobatum (L.) Schott., Wien. Zeitschr. 3: 72 (1829). Arum trilobatum L. A tuberous herb. Representative specimen: Putail, 03.03.06, Kanika 71 (DUSH).

\section{Lemnaceae}

Lemna perpusilla Torrey, Fl. N. York. 2: 245 (1843). A free floating, aquatic herb. Representative specimen: Putail, 03.03.06, Kanika 57 (DUSH). 


\section{Commelinaceae}

Commelina benghalensis L., Sp. Pl.: 41 (1753). Local nams: Kanchira, Dholpata. A slender herb. Representative specimen: Nabagram, 04.03.06, Kanika 112 (DUSH).

\section{Cyperaceae}

Cyperus compressus L., Sp. Pl. ed. 1: 46 (1753). A tufted annual herb. Representative specimen: Manikgonj, 02.03.06, Kanika 08 (DUSH).

C. diffusus Vahl, Enum. Pl. 2: 321 (1806). A perennial herb. Representative specimens: Putail, 27-12-06; Manikgonj Sadar, 10.09.06, Kanika 168 (DUSH).

C. distans L. f., Suppl. Pl.: 103 (1781). Local name: Panimalancho. A perennial herb. Representative specimen: Jaigir, 30.12.06, Kanika 271 (DUSH).

C. iria L., Sp. Pl. ed. 1: 45 (1753). Local name: Barachancha. An annual herb. Representative specimen: Nabagram, 27.12.06, Kanika 219 (DUSH).

C. michelianus (L.) Link., Hort. Bot. Berol. Descr. 1: 303 (1827). Scirpus michelianus L. An annual herb. Representative specimen: Putail, 03.03.06, Kanika 58 (DUSH).

C. rotundus L., Sp. Pl.: 45 (1753). Local name: Mutha ghas. A perennial herb. Representative specimen: Putail, 03.03.06, Kanika 72 (DUSH).

Diplacrum caricinum R. Br., Prodr. Fl. Nov. Holl.: 241 (1810). An annual herb. Representative specimen: Betila, 12.09.06, Kanika 203 (DUSH).

Fimbristylis acuminata Vahl, Enum. Pl. 2: 285 (1806). An annual herb. Representative specimen: Manikgonj, 02.03.06, Kanika 09 (DUSH).

F. cymosa R. Br., Prodr. Fl. Nov. Holl.: 228 (1810). A rhizomatous, perennial herb. Representative specimen: Nabagram, 04.03.06, Kanika 113 (DUSH).

\section{Poaceae}

Axonopus compressus (Sw.) P. Beauv., Ess. Agrost. 12: 154 (1812). Milium compressum Sw. A perennial, tufted grass. Representative specimen: Nabagram, 04.03.06, Kanika 94 (DUSH).

Bambusa balcooa Roxb., Fl. Ind. 2: 196 (1832). Local names: Barakbans, Balukbans, Valku, Balkua. A tall, densely caespitose bamboo. Representative specimen: Betila, 15.06.06, Kanika 132 (DUSH).

Chrysopogon aciculatus (Retz.) Trin., Fund. Agrost.: 188 (1820). Andropogon aciculatus Retz. A glabrous grass. Representative specimen: Nabagram, 04.03.06, Kanika 96 (DUSH).

Cynodon dactylon (L.) Pers., Syn. Pl. ed. 1: 85 (1805). Panicum dactylon L. Local name: Durba. A creeping grass. Representative specimen: Nabagram, 04.03.06, Kanika 120 (DUSH). 
Cyrtococcum accrescens (Trin.) Stapf in Hook., Ic. Pl.: sub t. 3096 (1922). Panicum accrescens Trin. An annual, scrambling grass. Representative specimen: Putail, 03.03.06, Kanika 73 (DUSH).

Dactyloctenium aegyptium (L.) P. Beauv., Ess. Agrost. Expl. P1.: 15 (1812). Cynosurus aegyptius L. A stoloniferous, annual or short-lived perennial grass. Representative specimen: Putail, 11.09.06, Kanika 186 (DUSH).

Echinochloa crus-galli (L.) P. Beauv., Ess. Agrost. 53: 161 (1812). Panicum crus-galli L. Local name: Bara shyama ghas. An annual or perennial grass. Representative specimen: Manikgonj Sadar, 02.03.06, Kanika 12 (DUSH).

Echinochloa stagnina (Retz.) P. Beauv., Ess. Agrost.: 161 (1812). Panicum stagninum Retz. An aquatic, perennial grass. Representative specimen: Nabagram, 04.03.06, Kanika 114 (DUSH).

Eleusine indica (L.) Gaertn., de Fruct. 1: 8 (1789). Cynusurus indicus L. Local name: Malanga kuri. An annual, tufted herb. Representative specimen: Manikgonj Sadar, 02.03.06, Kanika 24 (DUSH).

Hemarthria protensa Steud., Syn. Pl. Glam.: 359 (1854). Local names: Chalia, Chailla. An erect to decumbent grass. Representative specimen: Putail, 03.03.06, Kanika 75 (DUSH).

Hygroryza aristata (Retz.) Nees in Wight \& Arn., Edinburgh. New Philos. J. 15: 380 (1833). Pharus aristatus Retz. A floating grass. Representative specimen: Garpara, 28.12.06, Kanika 236 (DUSH).

Imperata cylindrica (L.) Reaeschel, Nom. Bot. ed. 3: 10 (1797). Lagurus cylindricus L. Local names: Chan, Chau, Sarkanb, Son, Ulukhar. A perennial, rhizomatous grass. Representative specimen: Manikgonj Sadar, 02.03.06, Kanika 26 (DUSH).

Oplismenus compositus (L.) P. Beauv., Ess. Agrost. 54: 168 (1812). Panicum compositum L. A perennial grass. Representative specimen: Nabagram, 04.03.06, Kanika 115 (DUSH).

Panicum montanum Roxb., Fl. Ind. 1: 315 (1820). A perennial, tufted grass. Representative specimen: Putail, 03.03.06, Kanika 59 (DUSH).

P. repens L., Sp. Pl. ed. 2: 87 (1753). A perennial, rhizomatous grass. Representative specimen: Jaigir, 30.12.06, Kanika 253 (DUSH).

Paspalum scrobiculatum L., Mant. 1: 29 (1767). Local name: Goicha. An annual grass. Representative specimen: Putail, 03.03.06, Kanika 61 (DUSH).

Saccharum spontaneum L., Mant. P1. 2: 183 (1771). Local name: Kash. A perennial, tall grass. Representative specimen: Jaigir, 30.12.06, Kanika 246 (DUSH).

Setaria palmifolia (Koen.) Stapf, J. Linn. Soc. Bot. 42: 186 (1914). Panicum palmaefolium Koen. Local name: Urodhan. A perennial grass. Representative specimen: Putail, 03.03.06, Kanika 74 (DUSH). 
Themeda quadrivalvis (L.) O. Kuntze, Rev. Gen. Pl. 2: 793 (1891). Andropogon quadrivalvis L. An annual herb. Representative specimen: Manikgonj Sadar, 02.03.06, Kanika 11 (DUSH).

Vetiveria zizanioides (L.) Nash in Small, Fl. Southeast U.S.: 67 (1903). Phalaris zizanoides L. Local names: Bena, Ghandhabena, Gandamul, Benamul. A rhizomatous, aromatic, perennial grass. Representative specimen: Manikgonj Sadar, 02.03.06, Kanika 26 (DUSH).

\section{Zingiberaceae}

Alpinia nigra (Gaertn.) Burtt., Notes Roy. Bot. Gard. Edinb. 35: 213 (1977). Zingiber nigrum Gaertn. Local name: Tara. A rhizomatous perennial herb. Representative specimen: Manikgonj Sadar, 02.03.06, Kanika 13 (DUSH).

Curcuma zedoaria (Christm.) Rosc. in Trans. Linn. Soc. Lond. 8: 354 (1807). Amomum zedoaria Christm. Local name: Shoti. A rhizomatous herb. Representative specimen: Nabagram, 27.12.06, Kanika 217(DUSH).

\section{Costaceae}

Costus speciosus (Koen.) Smith, Trans. Linn. Soc. London 1: 249 (1791). Banksea speciosa Koen. Local names: Gardong, Jongliphul. A rhizomatous herb. Representative specimen: Betila, 12.09.06, Kanika 204 (DUSH).

\section{Pontederiaceae}

Eichhornia crassipes (Mart.) Solms in A. DC., Mon. Phan. 4: 527 (1883). Pontederia crassipes Mart. Local name: Kachuripana. A free-floating herb. Representative specimen: Nabagram, 04.03.06, Kanika 95 (DUSH).

Monochoria hastata (L.) Solms. in A. DC., Mon. Phan. 4: 523 (1883). Pontederia hastata L.. An aquatic, emergent herb. Representative specimen: Putail, 03.03.06, Kanika 60 (DUSH).

\section{Liliaceae}

Asparagus acerosus Roxb., Fl. Ind. 2: 150 (1832). Local name: Shatamuli. A perennial, subscandent undershrub. Representative specimen: Betila, 15.06.06, Kanika 133 (DUSH).

Curculigo orchioides Gaertn., de Fruct. 1: 63, t. 13 (1788). Local names: Tali, Talamuli, Talura, Langtipata. A rhizomatous, perennial herb. Representative specimen: Manikgonj Sadar, 10.09.06, Kanika 170 (DUSH).

Molineria recurvata (Dryand.) Herbert, Amaryl.: 84 (1834). Curculigo recurvata Dryand. Local name: Satipata. A herb with tuberous rootstocks. Representative specimen: Nabagram, 04.03.06, Kanika 116 (DUSH).

\section{Smilacaceae}

Smilax zeylanica L., Sp. Pl. 2: 1029 (1753). Local name: Kumarilata. A prickly climber. Representative specimens: Garpara, 28.12.06, Kanika 237 (DUSH); Jaigir, 30.12.06, Kanika 220 (DUSH). 


\section{Dioscoreaceae}

Dioscorea bellophylla (Prain) J.O. Voigt ex Haines, For. Fl. Choto Nagpur: 530 (1910). Dioscorea nummularia var. belophyla Prain. Local name: Shora Alu. A perennial climber. Representative specimen: Nabagram, 04.03.06, Kanika 118 (DUSH).

D. bulbifera L., Sp. Pl. 2: 1033 (1733). Local name: Bon Alu. A large climber. Representative specimen: Manikgonj Sadar, 10.09.06, Kanika 171 (DUSH).

D. hispidia Dennst., Hort. Ind. Malabar.: 33 (1818). Dioscorea daemona Roxb. A twining climber. Representative specimen: Nabagram, 27.12.06, Kanika 215 (DUSH).

D. pentaphylla L., Sp. Pl. 2: 1032 (1753). Local name: Jhum Alu. A climber. Representative specimen: Manikgonj Sadar, 02.03.06, Kanika 27 (DUSH).

\section{Orchidaceae}

Vanda tessellata (Roxb.) Hook. f. ex G. Don in Loud., Hort. Brit.: 372 (1830). Epidendrum tessellatum Roxb. An epiphytic herb. Representative specimen: Putail, 03.03.06, Kanika 67 (DUSH).

\section{Acknowledgement}

The authors are grateful to Dr. M. Matiur Rahman, Former Director, Bangladesh National Herbarium for allowing to use herbarium facilities during the study. They also gratefully acknowledge the cooperation rendered by Mohammad Shah Alam.

\section{References}

Alam, M.K. 1995. Diversity in the woody flora of Sal (Shorea robusta) forest of Bangladesh. Bangladesh J. Forest Sci. 24(1): 41-51.

Alam, M.S., M.A. Hassan. and M.Z. Uddin. 2006. A Preliminary Checklist of the Angiospermic flora of Ghagotia Union under Kapasia Upazila in Gazipur District, Bangladesh. Bangladesh J. Plant Taxon. 13(2): 155-170.

Cronquist, A. 1981. An integrated System of Classification of Flowering Plants. Columbia University Press, New York, 1-1262.

Hooker, J.D. 1872-1897. The Flora of British India. Vols 1-7 (Ind. Repr. 1973). Bishen Singh Mahendra Pal Singh, Dehra Dun, India.

Hossain, A.B.M.E., S.A. Khan, and M.A. Islam. 1995. An inventory of plant diversity in relation with the ecology and environment of Jahangirnagar University. Vegetational composition and their taxonomic identity. Bangladesh J. Life Sci. 7 (1\&2): 95-103.

Ismail, M. and M.M.K. Mia. 1973. Studies on some deciduous Sal forests of Bangladesh. Ecology of Bangladesh Vegetation 2: 81-103.

Prain, D. 1903. Bengal Plants. Vols 1\&2. Indian Reprient 1981. Bishen Singh Mahendra Pal Singh, Dehra Dun, India. 1-1013.

Rizvi, S.N.H. 1969. Bangladesh District Gazeteers, Dacca, East Pakistan Govt. Press, Dacca.

Rashid, S. H., M.M Rahman and A.B.M.E. Hossain.1995. An inventory of the under growth resources in Chandra Sal forest at Gazipur, Bangladesh. Bangladesh J. Life Sci. 7(1\&2): 111-118. 
Rahman, M.O. and M.A. Hassan. 1995. Angiospermic Flora of Bhawal National Park, Gazipur (Bangladesh). Bangladesh J. Plant Taxon. 2 (1\&2): 47-79.

Ramzan, M. 2003. Manikgonj Sadar. in: Islam, S. (ed.), Banglapedia.Vol. 6. Asiatic Society of Bangladesh, Dhaka, $407 \mathrm{p}$.

Uddin, M.Z. and M.A Hassan. 2004. Flora of Rema- Kalenga Wildlife Sanctuary. IUCN Bangladesh Country Office, Dhaka, Bangladesh, 1-120.

(Received revised manuscript on 29 October 2013) 